\title{
STAR-FORMATION AND NUCLEAR ACTIVITY IN THREE
}

\section{GALAXIES WITH NUCLEAR RINGS}

\author{
THAISA STORCHI-BERGMANN* \\ Instituto de Fisica, UFRGS, C.P. 15051, P. Alegre, RS, Brasil \\ ANDREW S. WILSON* \\ Space Telescope Science Institute, Baltimore, MD, USA \\ and \\ JACK A. BALDWIN \\ Cerro Tololo InterAmerican Observatory, La Serena, Chile
}

We investigate two current problems in active galactic nuclei - the mode of fueling the putative black hole, and the question whether the circumnuclear regions have experienced unusual chemical processing - by studying the kinematics and chemical abundance of the gas in the nuclear region of galaxies with sites of ongoing star formation near the active nucleus. We discuss the results for three galaxies with nuclear rings: NGC1097 - for which we recently discovered broad double peaked $\mathrm{H} \alpha$ and $\mathrm{H} \beta$ emission from its LINER nucleus (Storchi-Bergmann, Baldwin \& Wilson 1993, ApJ 410, L11); NGC1672, which also presents a LINER nucleus; and NGC5248, a galaxy with a ring but no nuclear activity, used as a comparison. Narrow-band images obtained with the CTIO $1.5 \mathrm{~m}$ telescope were used to map the emitting gas. Longslit spectroscopy obtained with the $4 \mathrm{~m}$ telescope at high spectral resolution (at several positions over the nuclear region) was used to obtain the gas velocity field. In the two galaxies with LINER nucleus, the starforming rings are located in the turnover of the rotation curves, which show that the gas is rotating faster at the rings than farther out. We conclude that the rings are associated with inner Lindblad ressonances, which may be particularly effective in forcing gas inwards and fuelling the black hole (Wilson et al. 1986, ApJ 310, 121). From the emission line ratios the gas excitation is maped, and it is found that even in NGC5248, the nucleus presents a different excitation, suggesting a very mild LINER activity. Low-dispersion spectroscopy was also obtained in order to calculate the chemical abundances and compare the values obtained for the nuclear gas with those obtained for the HII regions in the ring and beyond the ring (when present). The goal is to check the results of recent studies (Storchi-Bergmann \& Pastoriza 1989, ApJ 347, 195; 1990, PASP 102, 1359), based on spectroscopy of the nucleus, which indicate an enhanced abundance of nitrogen, up to 5 times solar for the gas of the narrow line region of LINER and Seyfert 2 nuclei.

\footnotetext{
- Visiting Astronomer at the Cerro Tololo Interamerican Observatory, operated by AURA under contract with the National Science Foundation

T. J.-L. Courvoisier and A. Blecha: Multi-Wavelength Continuum Emission of AGN, 460. (C) 1994 IAU. Printed in the Netherlands. 